\title{
The Neuronal Theory in the Pathogenesis of Vitiligo: Past and Present
}

\author{
Mohammed S Al Abadie PhD FRCP \\ Consultant Dermatologist, Royal Wolverhampton NHS Hospitals. United Kingdom
}

Corresponding Author: Mohammed S Al Abadie PhD FRCP, Consultant Dermatologist, Royal Wolverhampton NHS Hospitals. United Kingdom.

Received date: August 16, 2021; Accepted date: August 28, 2021; Published date: September 10, 2021

Citation: Mohammed S Al Abadie. (2021) The Neuronal Theory in the Pathogenesis of Vitiligo: Past and Present. J. Dermatology and Dermatitis. 6(3); Doi: 10.31579/2578-8949/082

Copyright: @ 2021 Mohammed S Al Abadie, This is an open-access article distributed under the terms of The Creative Commons. Attribution License, which permits unrestricted use, distribution, and reproduction in any medium, provided the original author and source are credited.

\begin{abstract}
Vitiligo is a common skin disorder with an estimated prevalence of $1-2 \%$ of the world population. It is characterised by well demarcated milky white patches of depigmentation which have the tendency to enlarge and spread peripherally. The pathogenesis is not fully understood, a process leads and end in the loss of functional melanocytes from the epidermis. There are many proposed theories and hypotheses, none of which can fully explain its pathogenesis. However, lately it is believed that an overlap between some of these theories can present better understanding. This is to include the two long standing theories i.e., the autoimmune and the neuronal. The latter been proposed in 1959, initially based on clinical observations and studies, including animal, physiological, biochemical, embryonic, structural and electron microscopy. In 1994 new evidence emerged linking the neuronal theory to autoimmune mechanisms by demonstration of changes in Neuropeptides in particular Neuropeptide Y (NPY), in the skin at the active edge of vitiligo skin. This review paper highlights all evidence supportive of the neuronal theory in the pathogenesis of vitiligo past and present.
\end{abstract}

Key Words: Vitiligo; Neuronal Theory; Neuropeptide Y (NPY); Neuropeptides

\section{Introduction}

Vitiligo is a common idiopathic skin disorder w hich can affect 1-2\% from any population, although it is reported to be significantly higher in certain populations. Vitiligo is characterized by progressive circumscribed patches of depigmentation which have the tendency to enlarge and spread peripherally. Basically, this is due to the loss of functional melanocytes from the epidermis. The exact pathogenesis of vitiligo is still not fully understood, hence there are many theories and hypothesis been proposed including the autoimmune, Neuronal, melanocyte self-destruction, or defective adhesion, degenerative, composite and convergence. Non can explain all pathophysiology, however, lately it is believed that there is an overlap or link between some of these theories to fully understand the complete picture of vitiligo pathogenesis.

The Neuronal theory was first introduced by Lerner in 1959. He postulated the neuronal theory in the pathogenesis of vitiligo. $\mathrm{He}$ proposed that the nerve ending in the skin release substance which can be cytotoxic leading to the destruction of the Melanocytes. Initially it was thought this Catecholamines possibly others. The hypothesis was mainly based on theoretical proposition and many clinical observations (presented and discussed in this research).

The study presents for the first time the whole evidence supporting the neuronal theory from when it was first proposed into the most recent supporting evidence including the role of neuropeptides in the process of autoimmune events leading to the destruction of the melanocytes.

\section{The Neuronal theory}

Prior the mid-nineties the neuronal theory proposed by Lerner in 1959 [1], was mainly supported by several clinical observations and backed up by a number of embryonic, structural, biochemical, physiological and animal studies. In 1994 [2] the Neuronal theory been boosted with the discovery of the link of Neuropeptides and in particular Neuropeptide Y (NPY) in the pathogenesis of vitiligo. It was postulated that upon specific factors the release of such peptides can initiate a cascade of events where it can trigger the immune system in a process leading to the destruction of the melanocytes in a genetically predisposed individuals. Hence this has linked the neuronal and the autoimmune theories in the pathogenesis of vitiligo [2].

\section{Clinical Observations and Studies}


The concept of neuronal involvement in vitiligo is largely based on clinical observations. The most important of these is the finding of segmental distribution of depigmentation in some patients. Segmental does not mean dermatomal and usually several different dermatomes or a portion of one is involved. The depigmented segment has a nevoid appearance and usually, though not invariably, unilateral [3]. Each depigmented lesion stops abruptly at the midline [3].

However, vitiligo has on occasion been reported in a definite dermatomal location, eg confined to the distribution to the trigeminal nerve [1]. The symmetrical distribution of the lesions also gives important evidence for neuronal involvement. In a patient with transvers myelitis paralyzed from the waist down, hypopigmentation was not spared in the areas below the level of cord damage, and vitiligo lesions only developed on the face [4] and upper portion of the body, ie the neck, axillae, arms and hands [1]. This distribution would be unusual for a patient with generalized vitiligo. Furthermore, it was unusual that the koebner reaction did not result in the surgical scar when hysterectomy was carried out on this patient. Vitiligo has also been reported following peripheral nerve injury [5], in a child with viral encephalitis [6], and in patients with multiple sclerosis who had Horner's syndrome [7]. Spontaneous re-pigmentation of extensive vitiligo has been observed in a diabetic patient who developed sever diabetic neuropathy [8]. A further supportive case is that of a patient who developed a traumatic injury to the right brachial plexus, who exhibited ipsilateral macules of vitiligo at the level of the upper arm, chest, buttock, sacrum and scrotum [9]. The author suggested the phenomenon of sympathetic repercussion has resulted in the widespread leukoderma and that irritation of the sympathetic nerve endings of the $\mathrm{C} 8$ and $\mathrm{D} 1$ roots has induced a series of reflexes which resulted in depigmentation exceeding the area innervated by the brachial plexus.

Surgical transplant studies have also given support to the neuronal concept of vitiligo. Haxthausen [10] transplanted skin from pigmented to non-pigmented area and vis versa. He showed that transplanted skin always taken the characteristics of the area into which the graft is placed but that this only happens after the local innervation has been reestablished. Others have made similar findings [11].

Lerner (1) postulated that vitiligo probably resulted from increased output at the peripheral nerve endings of a substance that lightens melanocytes. This view is shared by Panja [12] who suggested that melanocytes become devoid of their function as a consequence of dysfunction or damage to autonomic nerve components. He regarded vitiligo as "trophoneurosis" affecting the adreno-autonomic system. The link of vitiligo to emotional trauma and stress has added an extra value and strength to the clinical observation in support of the neuronal theory.

\section{Animal Studies}

The neuronal influence on the skin color of certain animals is well known. In the lower animals there is direct adrenergic response and the melanophores were found to have direct innervations [13, 14]. In fish electrical stimulation of cutaneous nerves causes blanching, and sectioning the nerve cause darkening. Pigment cells of fish, amphibians and reptiles have alpha-and/or beta- adrenergic receptors. Alpha stimulation lightens the skin by the aggregation of melanosomes, and stimulation disperses granules to cause darkening [15]. In frogs, the pigment cells are not innervated directly, yet these cells, like those in lizards respond to pharmacologically active adrenergic and cholinergic chemicals as well as their blocking agents [16] Parker [17] examined the dark dorsal and pale ventral skin of the cat fish (Ameirus) and found a higher concentration of acetylcholine in the dark portions compared to the pale areas. Furthermore, Fabian [18] showed an alteration of cutaneous pigmentation when denervating the skin in guinea pigs. Acetylcholine, noradrenaline, adrenaline and melatonin were found to have a lightening effect on the dermal melanocytes in the frog and in other marine animals
[1]. In a study on inbred black rats white hair after the injection of Adrenaline / Epinephrine [19].

\section{Physiological and Biochemical studies}

The clinical observation of a link between the nervous system and vitiligo initiated many physiological and biomedical studies. Chanco-Turner and Lerner $[20,21]$ measured the surface temperature, sweat production and bleeding time in vitiliginous skin and compared it to normal. Their study showed the temperature and sweat production vitiliginous skin and compared to normal. Their study showed that the temperature and sweat production in vitiliginous skin were increased, and the bleeding time was prolonged. However, injection of acetylcholine and adrenaline (at a concentration of $1 ; 10,000)$, as well as of histamine $(1: 100,000)$ into the vitiliginous and normal skin did not reveal any difference in the wheal and flare reaction or, following injection of acetylcholine, in sweat production [20]. Likewise, sweat production after the injection of adrenaline and the triple response to histamine was the same in both vitiliginous areas exhibited either excessive cholinergic activity or relatively insufficient adrenergic tone [20]. Other studies have also shown abnormalities in such parameters as blister to the injection of adrenaline [22]. Dutta also concluded that vitiliginous skin had an impaired sympathetic adrenergic tone. Koga [23], studying patients with segmental or generalized vitiligo, found the reaction to injections of physostigmine, an inhibitor of cholinesterase, was far higher in segmental vitiligo patients compared to that in patients with generalized vitiligo. The treatment of segmental vitiligo with nialamide, a monoamine oxidase inhibitor, produced re pigmentation in 16 out of 28 patients (55\%) [23]. The conclusion from this study was that the depigmented skin of patients with early stage segmental vitiligo exhibits adrenergic hypotonia; in a later stage, hyperactive adrenergic response is noted.

The axon reflex sweat secretion test with nicotinic tartrate $(1: 100,000$ dilution) and the surface temperature were reported to be alerted in vitiliginous skin compared to normal [24]. Altered autonomic function in vitiliginous skin has also been found $[1,25,26]$. In Contrast, another study showed no difference in cutaneous sensation and perspiration between vitiliginous and normal skin: intradermal injection of acetylcholine $(1: 60,000)$ showed the same degree of sweating in both types of skin [27].

Biochemical studies have showed that pathways for the metabolism of the amino acid tyrosine and its oxidative derivative, dopa, are the substrates for both melanin and the neurotransmitters adrenaline and non-adrenaline [3].

\section{Embryonic, Structural and Electron Microscopy Studies}

Melanocytes and nerve cells are both derived from the neural crest and melanocytes are thought to migrate towards the epidermis at the third trimester [1, 28, 29]. Melanoblast migrates from the dorsal area of the neural tube to the basal layer of the epidermis, and hair bulb, inner ear, uvea and meninges [30]. This may also explain how the outer root sheath acting as a source of reservoir for repigmentation in Ultra Violet therapy in vitiligo. In segmental vitiligo a degree of sympathetic verve disfunction was found [31]. The production of pigment and melanocytic development are directly regulated by local sympathetic adrenergic innervation, an increased activity of which has been shown in vitiligo lesions [32]. In vitiligo, a direct anatomical contact between nerve fibers and melanocytes has been seen [33].

Shao Chan-Gen [34], using a silver impregnation technique, described dystrophic changes among the nerve trunks and terminals in vitiligo lesions. He reported a significant difference in intensity of staining of nerve fibers in lesions with a very long duration. Furthermore, an electron microscopic study by Breathnach [35] showed degenerative and regenerative changes in the terminal regions of the nerve supply, and in 
central and marginal areas of vitiliginous lesions. Other studies have also demonstrated degenerative changes in the nerves of vitiligo lesions [36, 37]. Further Electron Microscopy Studies on skin peripheral nerves in vitiligo show significant changes. An increase thickness of the basement membrane of Schwann cells in $75 \%$ of patients compared to normal skin control, $50 \%$ of the abnormal dermal nerves in vitiligo showed minor axonal damage [38].

Morphological and functional link was also found between the Melanocytes and nervous system [39]. In an acute active vitiligo skin, significant degeneration was found in dermal nerves. Complete degeneration of the nerves seen in $41 \%$ of patients, $38 \%$ showed partial degeneration. Nerve ending showed similar pattern [40].

\section{Emotional Trauma and Stress}

The onset of vitiligo sometimes is associated with severe emotional trauma such as the death of parent or spouse, the loss of a job, financial ruin, or a sever fright, and extension of the disease often occurs when the patient experiences great stress. Supportive case histories include that of a young girl, nearly struck by a gunfire in a war who became suddenly aware of having vitiligo [41]. Another is that of a hysterical woman who complained of pain in the back of right thigh and who developed urticaria and vitiligo after the insertion of electromyographic needles [42]. Clinical impression as well as patients' views often relate the disease's initial attack or subsequent flare-up to severe stressful event, usually emotional in character, such as marital quarrel, death of loved one, tension at work or a frightening accident $[1,43,44]$. In one study $69 \%$ of the patients either claimed an emotional upset preceded their vitiligo or attributed their disease to nervousness, an accident, illness, an operation or parturition [1]. Acute vitiligo has been reported after an emotionally traumatic event [29]. Furthermore, vitiligo is also associated with severe emotional trauma such as the death of a parent or spouse, the loss of a job, financial ruin, or a sever fright, and extension of the disease often occurs when the patient experiences great stress. Supportive case histories include that of a young girl, nearly struck by gunfire in a war, who became suddenly aware of having vitiligo [41]. 30\% of patients reported significant emotional and stressful life events preceding the initial onset of the vitiligo with $39 \%$ report flare ups with stress [1]. Vitiligo patients mentioned personal problems in $47 \%$ of cases on third were related to exams, and $31 \%$ of cases were related to jobs or financial problems [45]. On the other hand, vitiligo can have psycho-social impact on sufferers $[46,47,48]$. The prevalence of vitiligo is reported to be higher in psychosis clinics rather that in dermatology clinics [49]. Vitiligo is said to typically occur in tense, emotionally liable people [1,21].

\section{Nerve Growth Factor (NGF)}

Nerve Growth Factor (NGF) release by keratinocytes was found to have a significant effect on melanocytic migration [50]. In skin NGF has an important role in the maintenance of sympathetic nerve fibers [51]. The significant upregulation in expression of p75 NGFr in vitiligo skin and the increasing evidence that NGF plays an important role between the nervous and immune systems, suggesting a potential role in the destruction of melanocytes in vitiligo patients [52]. Liu [53] showed a tendency for a reduction in the number and intensity of low affinity (p75) nerve growth factor receptor immuno-reactive (NGFr-IR) basal keratinocytes in involved vitiliginous skin compared with controlled skin, while the number of NGFr-IR nerve fibers was significantly increased [53].

\section{Neuropeptides}

In 1994 breakthrough research in Sheffield, using semi-quantitative immuno-histochemical methods, linked the neuronal theory to autoimmune mechanisms by the demonstration of changes in neuropeptides, especially neuropeptide $\mathrm{Y}$, in the skin at the active edge of vitiligo macules [2]. The authors proposed that neuropeptides, notably neuropeptide $\mathrm{Y}$, release at the epidermal nerve ending release adjacent to melanocytes, provoke a local immune reaction leading to melanocyte destruction in predisposed individuals. Subsequent research has bolstered a role for neuropeptides and the neuro-immunological process in the causation of vitiligo in particular Neuropeptide Y (NPY) and possible role for others $[51,54,55,56]$. The link of neuropeptides with catecholamines is well established [4]. The increase of catecholamines in vitiligo can contribute to the destruction of melanocytes by direct cytotoxicity or indirectly by causing severe vasoconstriction as a result of the activation of alpha receptors of the skin arterioles. This can lead to sever hypoxia leading to the production of toxic oxygen radicals and that can lead to the destruction of the melanocytes [57]. In vitiligo patient's catecholamines neurotransmitters has an impaired metabolism. Plasma level of norepinephrine was significantly higher when compared to control [58]. Investigation of personality structures in patients with vitiligo and a possible association with impaired catecholamine. Norepinephrine and monoamine oxidase activities was also found to be increased in keratinocytes cultured from lesional and non lesional skin from vitiligo patients [59]. The level of Norepinephrine (NE), Normetanephrine (NMN), and their metabolites 3-methoxy-4-hydroxyphenylglycol (MHPG), Homovanillic acid (HVA), were found to be significantly higher in Vitiligo patients at the active phase of the disease [60] Catecholamine was also found in elevated in serum and urine of vitiligo patients $[61,62]$. Neuropeptide Y (NPY), is found to be co-localized and released with direct link with norepinephrine from nerve fibers once stimulated. Furthermore, it can modulate the release and the effect of norepinephrine on the sympathetic nervous system [4)]. Dopamine is another precursor of catecholamines. Its plasma level was found to be significantly higher in vitiligo patients compared to control [63]. This can induce apoptosis of the melanocytes in vitiligo, possibly linked to stress [61], in association with the increase of sympathetic nerve stimulation triggered by NPY inducing vasoconstriction during stress [25], leading to the induction of a local immunological reaction [64], a process which can end up with the destruction of the melanocytes.

Significant elevation of plasma and tissue fluid level of NPY was found in vitiligo patients. The level of NPY in the patients with vitiligo of generalised-local and segmental vitiligo were found to be significantly higher than normal control. In both local and segmental type, the levels in progressive stage were significantly higher than those in stable stage [55].

More recently, it was concluded that Neuropeptides and nerve growth factors are critical regulators in of emotional responses participating in the onset and development of vitiligo in certain predisposed individuals. Direct cytotoxicity to melanocytes and the initiation and propagation of local and systemic immune or inflammatory reaction; include a specific adaptive immune response leading to the destruction of melanocytes which can be triggered by the following neurogenic factors i.e. NPY, CGRP, Catecholamines, NGFs and NGF receptors [51].

Centrally Neuropeptide Y (NPY), can act as a neuro-endocrine integrator where it links the central and peripheral nervous system by linking the hypothalamus, pituitary and gonadal axis [65]. The reduced level of NPY in brain central amygdale lead to anxiety [66], while on the other hand NPY is also involved in stress response and mood swings proved by the studies showing the increase of (NPY) expression and release in the brain, indicating this can be directly involved with the emotion and mental stress [67]. Subsequently, emotional and mental stress can play a significant role in the production of cytokines and reactive oxygen spices (ROS), by triggering an inflammatory response initiated by antigen-presenting cells (APC), and Polymorphonuclear leukocytes (PMN). The process may well lead to the destruction of the melanocytes [51]. 
It is thought that Neuropeptides eg (NPY) has a direct interaction with the $\mathrm{T}$ cells, triggering events lead to the induction of Cytokines secretion phenotype [68, 69]. NPY, can affect the functions of the cells of the adaptive and innate immunity. In this respect, NPY is known to modulate immune cell trafficking, T helper cell differentiation, cytokine secretion, natural killer cell activity, phagocytosis, and the production of oxygen species. The specific Y receptors have been found in immune cells, and their expression is amplified upon immune stimulation [64].

Another Neuropeptide ie Calcitonin Gene Related Peptide (CGRP) which was also found to have a link between the nervous and immune system. It can be involved in chronic inflammatory responses which can trigger specific adaptive immune responses leading to the destruction of the melanocytes in general [51]. A role for CGRP release from the sympathetic nerve endings in the skin triggered by emotional stress and anxiety is also proposed in the distraction of the melanocytes by triggering a local and systemic inflammation by direct activation of keratinocytes, Langerhans cells, and glial cells in the skin. CGRP can also trigger the production of Catecholamines by stimulating hypothalamus-pituitaryadrenal axis [51, 70, 71]. CGRP nerve fibers were found to be significantly increased in the epidermis and papillary dermis involved vitiligo skin compared with controlled skin $[54,53]$. Other neuropeptides role in the pathogenesis of vitiligo is not clear and that include, Vasointestinal Peptide (VIP) questioning its presence at vitiligo skin [2], or the effects on the immune cells [72, 73].

\section{Conclusion}

It is proposed that Neuropeptides release provoking local immune reaction leading to the destruction of Melanocytes, in a genetically predisposed individual. Such link was confirmed where an increase level of Neuropeptide Y (NPY) in a specific population with vitiligo was found to be associated with genetic variant of interleukin 1B (IL1B). Further work is needed to explore the relationship of neuropeptides and the process of keratinocyte stimulation by interferone- $\gamma$ producing CXCL9/10 leading to the recruitment of the melanocytotoxic T cells. Blocking such process by Janus kinase (JAK)-1 inhibitors might be the key to stop the cascade of events leading to the destruction of the melanocytes.

\section{References}

1. Lerner AB. (1972). Vitiligo. Progressin Dermatology. 6:1.

2. Al Abadie MS, Senior HJ, Bleehen SS and Gawkrodger DJ. (1994). Neuropeptides and Neuronal Marker Studies in Vitiligo. British Journal Dermatol. 131: 160-165.

3. Nordlands JJ. (1986). Vitiligo. In: Pathogensis of skin diseases. Eds. Thiers BH and Dobson RL. Churchill Livingstone, New York. P 99.

4. Han S, Yang CL, Chen X, Naes L, Cox BF, Westfall T. (1998). Direct evidence for the role of neuropeptide $\mathrm{Y}$ in the sympathetic nerve stimulation-induced vasoconstriction. Am J Physiol. 274:290-294.

5. Arnozan L. (1922). Vitiligo avec troubles nerveux sensitifs et sympathetiques: l'origine sympathique du vitiligo. Bull Soc. Fr. Dermatol, Syphili. 29:338.

6. Nellhaus G. (1970). Acquired unilateral vitiligo and poliosis of the head and subacute encephalitis with partial recovery. Neurology: 323, 809.

7. Jonesco-Siseti MM, Vasileco N, and Palade G. (1937). Sclerose en plaques avec syndrome de Claude Bernard-Horner et vitiligo. Bull.Mem.Soc.Hosp.Paris. 53:941

8. Lerner AB. (1972). Vitiligo. Progressin Dermatology. 6:1.

9. Costa V. (1961). Leukoderma patches in the course of traumatic paralysis of the brachial plexus in a subjects with insular cavities. Dermato-Venerologica. 2:161.
10. Haxthausen H. (1947). Studies on the pathogenesis of morphoea, vitiligo and acrodermatitis atrophicans by means of transplantation experiments. Acta Derm Venereol (Stockh.). 27:352.

11. Spencer GA, and Tolmach JA. (1952). Exchange grafts in vitiligo. J Invest Dermatol. 19:1.

12. Panja RK. (1977). Etiology of vitiligo-a problem. Ind J Dermatol Vener Lepr. 43:18.

13. Novales A. (1960). Response of tissue-cultured embryonic new melanophores to epinephrine. Trans Am Micr Soc. 79: 25-33.

14. Jacobowitz DM, laties AM. (1968). Direct adrenergic innervations of teleost melanophore. Anat Res. 162:501-504.

15. Mosher DB, Fitzpatrick TB, Ortonne JP, and Hori Y. (1987). Abnormalities of pigmentation. In: Dermatology in general practice medicine ( $3^{\text {rd }}$ edition). Eds. Fitzpatrick TB, Eisen AZ, Wolff K, Freedberg IM, and Austen KF. McGraw-Hill, New York. p 794.

16. Lerner AB. (1971). On the etiology of vitiligo and grey hair. Am J Med. $51: 141$.

17. Parker GH, Welsh JH, and Hyde JE. (1945). The amount of acetylcholine in the dark skin and the pale skin of the cat fish. Proc Nat Acad Sci. 31:1.

18. Fabian G. (1951). The spread of black pigment of the denervated skin of the guinea pig. Acta Bilo Acad Sci Hung. $4: 471$.

19. Shelly WB, \& Ohman S. (1969). Epinephrine induction of white hair in ACI rates. J Invest Dermatol. 93: 155-158.

20. Chanco-Turner ML, and Lerner AB. (1965). Physiological changes in vitiligo. Arch Dermatol. 91:390.

21. Lerner AB. (1966) Sympathectomy and grey hair. Arch Dermatol. 93:235.

22. Dutta AK. (1972). Non-nervous vascular reactions in vitiligo patches. Indian J Dermatol. 17:29.

23. Koga M. (1977). Vitiligo: A new classification and therapy. Brit J Dermatol. 97:255.

24. Kaur A and Sarin RC. (1988). Study of neurotropic influences in vitiligo. Ind J Dermatol. 33(3):33.

25. Rothman S, Rubin L, and Houston M. (1943). Vitiligo. Arch Dermatol Syph. 48:400.

26. Ono T. (1963). Pathophysiological studies in vitiligo vulgaris. Jpn J Dermatol. 73:176.

27. Gopinathan T. (1965). A study of the lesion of vitiligo. Arch Dermatol. 91:397.

28. Breathnach AS and Wyllie LM. (1965). Electron microscopy of melanocytes and langherhans cells in human foetal epidermis at fourteen weeks. J Invest Dermatol. 45:51.

29. Lerner AB, and Nordlund J. (1978) Vitiligo, what is it? Is it important? J Am Acad Dermatol. 239:1183

30. Hsin-Su Ya. (2002). Melanocytes destruction and repigmentation in vitiligo. A model for nerve cells damage and regrowth. Journal of Biomedical Science. 9(6):564-573.

31. Wu CS, Yu HS, Change HR, et al. (2000). Cutaneous blood flow and adreno-receptor response increase in segmental-type vitiligo lesions. J Dermatol Sci. 23: 53-62.

32. Schalleruter KU et al. (1993). Arch Dermatol Dermatol Res. 285:216-20.

33. Morohashi M, Hashimoto K, Goodman F Jr, Newton E, and Rist T. (1977). Ultrastructral studies of vitiligo. VogtKoyanaagi-Harada Syndrome and incontentia pigment achromians. Arch Dermatol. 113:755.

34. Shao-Chan Gen. (1959). Changes of the neuro receptor apparatus in vitiligo. J Invest Dermatol. 33:20. 
35. Breathnach AS, Bor S, and Wyllie LM. (1966). Electron microscopy of peripheral nerve terminals and marginal melanocytes in vitiligo. J Invest Dermatol. 47:125.

36. Mori H. (1964). Neurohistological studies in vitiligo vulgaris skin with special reference to vegetative nerves. Jpn J Dermatol. 74:411.

37. Nozaki T. (1976). Histochemical and electromicroscopic studies of peripheral nerve terminals and clinical studies in vitiligo vulgaris. Jpn J Dermatol. 86:1

38. Al Abadie MS, Warren MA, Bleehen SS and Gawkrodger DJ. (1995). Morphological Observations on the Dermal Nerves in Vitiligo and Ultrastructural Study. Int. J Dermatol. 34(12): 837-840.

39. Hara M and Toyoda M, Yaar M et al. (1996). Innervation of melanocytes in human skin. J Exp Med. 184:1385 -1395.

40. Gokhale B and Mehta L. (1983). Histopathology of Vitiliginous skin. Int J Dermatol. 22:277-280.

41. Breathnach AS. (1963). In discussion of Induced alterations of vitiliginous skin. Ann NY Acad Sci. 100:58.

42. Walpin LA, Reiss F. (1966). Urticaria changing to vitiligo provoked by electromyographic examination. Acta derm Venerol (Stockh). 46:242.

43. Dutta AK, and Manndel SB. (1969). A clinical study of 650 vitiligo cases and their classifications. Indian J Dermatol. 14:103.

44. Silverton N. (1972). Vitiligo in immigrants (Letter). Lancet. 1:200.

45. Liana Manolcache and Vasile Benea. (2007). Stress in patients with alopecia areata and vitiligo. JEADV. 21:921-928.

46. Al Abadie MS, Kent GG. (1996). Factors Affecting responses on the Dermatology Life Quality Index among Vitiligo Suffers. Clinical \& Experimental Dermatol. 21: 330-333.

47. Kent GG, Al Abadie MS. (1996). Psychological Effect Vitiligo. A Clinical Instant analysis. J Acad Dermatol. 35:895898.

48. Al Abadie MS, Ashraff F, Al Abadie D. (2019). Impact of childhood Vitiligo on Self Esteem and Quality of Life in Adulthood. Archives of Dermatology and Skin Care. 2(2): 0106.

49. Obermayer ME. (1955). Psychoses and disorders of the skin: Psychocutanous Medicine. Springfield, Illinois. p 404.

50. Pincelli C \& Yaar M. (1997). Nerve Growth Factor: Its significance in cutaneous biology. J Invest Dermatol Symp Proc. 2:31-36.

51. Levi-Montalcini R, Skaper SD, Dal Toson R et al. (1996). Nerve Growth Factor: From neurotrophin to neurokine. Trends Neurosci. 19: 514-520.

52. Yu R, Huang Y, Zhang X and Zhou Y. (2012). Potential Role of Neurogenic Inflammatory factors in the pathogenesis of Vitiligo. J Cut Med and Surg. 16 (4):230-244.

53. Liu P, Bondesson L, Lontz W, \& Johansson O. (1996). The Occurrence of cutaneous nerve endings and neuropeptides in vitiligo vulgaris: A Case control study. Archives of Dermatological Research. 288 (11):670-675.

54. Lazarova R, Hirstakieva E, Lazarova N \& Shani J. (2000). Vitiligo Related Neuropeptides in Nerve fibers of the skin. Archives of Physiology and Biochemistry. Vol 108, No3 pp 263-267.

55. Hirstakieva E, Lazarova R, Lazarova N, et al. (1999). Markers for Vitiligo related neuropeptides in human skin nerve fibers. Acta Med Cro. 54:53-57.
56. Tu C, Zhad D and Lin X. (2001). Levels of Neuropeptide-Y in the Plasma and Skin tissue fluids with Vitiligo Journal of Derm Sci. 27 (3): 178-182.

57. Morrone A, Picardo M, De Luca C, Terminali O, Passi S, Ippolito F. (1992). Catecholamines and vitiligo. Pigment Cell Res. 5:65-69.

58. Salzer BA and Schallreuter KU. (1995). Investigation of personality structure in patients with vitiligo and a possible association with impaired catecholamine metabolism; Dermatology, Basel, Switzerland). (2): 109-115.

59. Schallreuter KU, Wood JM, Pittelkow MR et al. (1996). Increased monoamine oxidase A activity in the epidermis of patients with vitiligio. Arch Derm Res. 288:14-18.

60. Cucchi ML, Frattini P, Santagostino G and Orecchia G. (2000). Hiher Plasma Catecholamine and Metabolite levels in the early phase of Vitiligo. 13: 28-32.

61. Cucchi MI, Drattini P, Santagostino G et al. (2003). Catecholamines increase in the urine of non-segmental vitiligo especially during its active phase. Pigment cells Res. 16:111116.

62. Chu CY. Liu YL, Chiu HC and Jee SH. (2006). DopamineInduced Apoptosis in human melanocytes involve generation of reactive oxygen species. Br J Dermatol. 154 (6):1071-1079.

63. Kotb El-Sayed MI, Abd El-Ghanny AA, Mohamed RR. (2018). Neural and Endocrinal pathoblochemistry of vitiligo: comparative study for a hypothesized mechanism. Front Endocrinol (Lausanne). 9:197.

64. Dimitrijevic M and Stanojevic S. (2013). The intriguing mission of neuropeptide $\mathrm{Y}$ in the immune system. 45:41-53.

65. Hill JW, Elmquist JK, and Elias CF. (2008). Hypothalmic pathways linking energy balance and reproduction. Am J Physiol Endocrinol Metabol. 294:827-823.

66. Pandey SC, Zhang H, Roy A, Xu T. (2005). Deficient in amygdaloid cAMP-responsive element-binding protein signaling play a role in genetic predisposition to anxiety and alcoholism. 115:2762-2773.

67. Morales-Medina JC, Dumont Y, Quirion R. (2010). A possible role of neuropeptide $\mathrm{Y}$ in depression and stress. Brain Res. 1314:194-205.

68. Song C, Leonard BE. (1998). Comparison between the Effects of Sigma receptor ligand Jo 1784 and Neuropeptide Y on immune functions Eur J Pharmacol. 345:79-87.

69. Levite M. (1998). Neuropeptides, by direct interaction with $T$ Cells, induce Cytokine secretion and break the com went to a distinct $\mathrm{T}$ helper phenotype. Proc Nat Acad Sci USA. 95:12544-12549.

70. Vause CV, Durham PL. (2010). Calcitonin-gene - related peptide differentially regulates gene and protein expression in trigeminal glia cells: findings from array analysis. Neurosci Lett. 473:163-167.

71. Dallos A, Kiss M, Polyanka H, et al. (2006). Effect of the neuropeptides Substance P, Calcitonin gene-related peptide, Vasoactive intestinal polypeptide and inflammatory cytokines in cultural human keratinocytes Neuropeptides. 40: 251-263.

72. Ganea D, Hooper KH, Kong W. (2015). The neuropeptide VIP: direct effects on immune cells and involvement in inflammatory and autoimmune diseases. Acta Physiol (Oxf). 213: 442-452.

73. Martins C, Darrigade AS, Jacquemin C et al. (2020). Phenotype and function of circulating memory $\mathrm{T}$ cells in human vitiligo. Br J Dermatol. 183: 899-908. 
(c) (P)

This work is licensed under Creative

Commons Attribution 4.0 License

To Submit Your Article Click Here: Submit Manuscript

DOI: $10.31579 / 2578-8949 / 082$
Ready to submit your research? Choose Auctores and benefit from:

* fast, convenient online submission

* rigorous peer review by experienced research in your field

* rapid publication on acceptance

* authors retain copyrights

* unique DOI for all articles

* immediate, unrestricted online access

At Auctores, research is always in progress.

Learn more auctoresonline.org/journals/dermatology-and-dermatitis 